\title{
Research on Characteristics of Hysteresis in Accordance with the Oil Line of Clutch System
}

\author{
Hyun-sik Kim ${ }^{1}$, Sung-mo Yang ${ }^{2, *}$ and Hyang-woo Kim ${ }^{3, *}$ \\ ${ }^{1}$ Department of Mechanical System Engineering, Chonbuk National University, Jeonju, 54896, South Korea \\ ${ }^{2}$ Department of Mechanical System Engineering, Chonbuk National University, Jeonju, 54896, South Korea \\ ${ }^{3}$ Department of Automotive Plant Industrial Engineering, Hanyeong College, Yeosu, 59720, South Korea \\ *Corresponding author
}

\begin{abstract}
This thesis conducted a comparison analysis via an experiment on clutch pedal effort hysteresis response characteristic in accordance with oil line characteristic which makes up the drive system in automobile clutch. Clutch drive system experiment Jig was composed and used in comparison analysis of response characteristic in accordance with change in length of oil line possessing length of $4 \mathrm{~m}$ and $8 \mathrm{~m}$ and response characteristic in accordance with change in material using $8 \mathrm{~m}$ length oil line possessing steel material, plastic material and rubber material with equivalent size data. The oil pressure applied to the oil line during the experiment was fixed at 8 bar and the applied pedaling time was $0.3 \mathrm{sec}$ and $1.0 \mathrm{sec}$. The result of the experiment showed the change in response characteristic aspect in accordance with oil line length change in clutch drive system was inadequate and was able to find out that the chance in response characteristic in accordance with oil line material change possessed the highest hysteresis response characteristic when steel material was applied. Through this study, we were able to understand the aspect of response characteristic change in hysteresis of clutch pedal effort in accordance with the characteristic change which makes up the oil line that composes clutch drive system.
\end{abstract}

Keywords-clutch system; hysteresis; oil line length; oil line material; pedal load

\section{INTRODUCTION}

The clutch applied in automobile is used to transmit the rotational power which is generated from the engine of the automobiles to the drive shaft and is a shaft coupling device which allows for cut off of the transmitting of electric power during transmits of rotary motion of the axis to the transmission axis. It is an essential process used during the speed control of power transfer unit, as it is capable of stopping the driven shaft without stopping the rotary movement if main driving axle connected to the motor during the change in speed of an automobile. Generally, the structure of automobile manual clutch uses auxiliary devices that converts oil pressure and air pressure into working fluid in its path of being transmitted from the clutch pedal to the clutch cover but these auxiliary devices cause change in clutch response characteristic and ultimately these changes in turn has a negative effect of increasing fatigue in a driver [1]. The clutch system operates by receiving the distribution from the oil pressure and air pressure device which is a clutch auxiliary device in addition to the applied force to the pedal when the driver steps on the clutch pedal. A study regarding an accurate hysteresis response characteristic between such pedal strength and the final pedal effort is necessary, and for the same there is also a need for an accurate analysis regarding auxiliary device and response characteristic which composes clutch system.

In this thesis, we constructed clutch drive experimental device and compared and analyzed clutch pedal effort hysteresis response characteristic in accordance with oil line characteristic change which makes up the clutch oil pressure device through an experiment.

\section{ClutCh System THEORY}

\section{A. Clutch System}

Generally the clutch drive system is structured to operate the clutch booster using the pressure of the fluid applied to the master cylinder and through this, it passes through release fork and diaphragm spring and finally runs the pressure plate of the clutch cover. Figure I. displays the schematic diagram of clutch drive system [1].
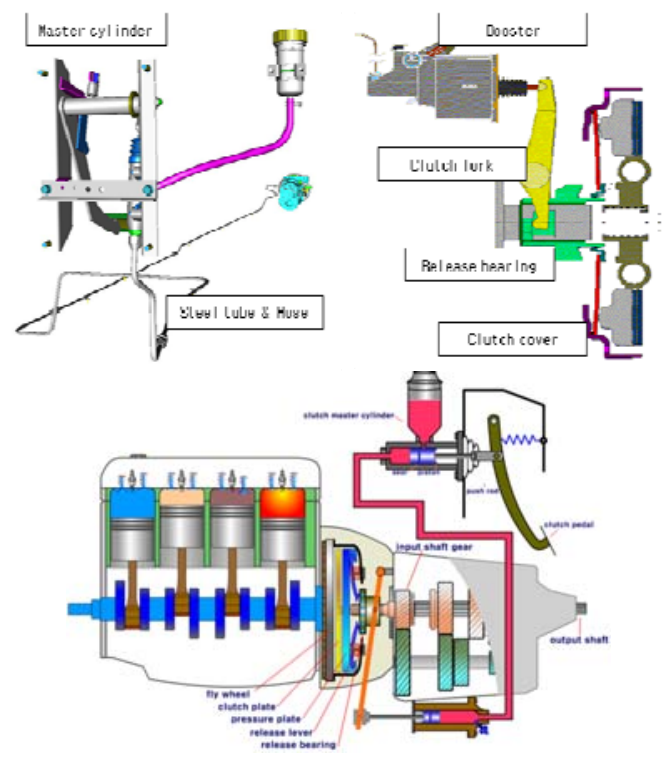

FIGURE I. CLUTCH SYSTEM SCHEMATIC 


\section{B. Theory of Test}

For the purposes of mathematical modeling of clutch drive system, equalization of each auxiliary device regarding mass, damping, hardness was done and this was reconstructed as the general system model. Figure II. displays mathematical modeling of clutch system performance and the variables used here are $P_{c}$ is inner line pressure, $X_{m}$ and $X_{r}$ is cylinder displacement, $X_{p}$ is push rod displacement, $\beta$ is bulk modulus, $V$ is oil pressure volume, $M_{m}$ and $M_{r}$ is piston mass, $b_{m}$ and $b_{r}$ is viscosity coefficient, $k_{m}$ and $k_{r}$ is return spring invariable, $k_{p}$ is push rod spring invariable, $A_{m}$ and $A_{r}$ is piston area, $f_{m}=k_{p}\left(X_{p}-X_{m}\right)$ is load spring capacity and $f_{d}=k_{l}\left(X_{l}-X_{r}\right)$ is load spring capacity. The interaction formula of these mathematical modeling are as follows.

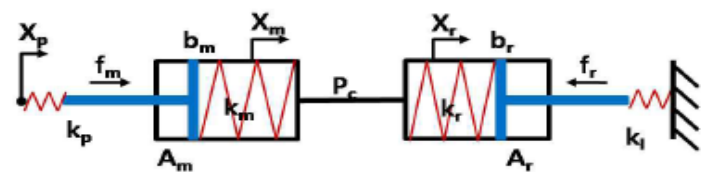

FIGURE II. CLUTCH SYSTEM MATHEMATICAL MODELING

$$
\begin{aligned}
& \dot{P}_{c}=\frac{\beta}{V}\left(A_{n p} \dot{x_{n}}-A_{\mathrm{r}} \dot{x}_{\mathrm{r}}\right)
\end{aligned}
$$

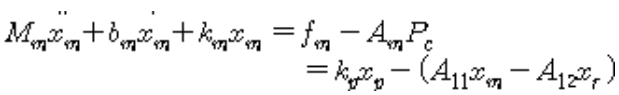

$$
\begin{aligned}
& M_{\mathrm{r}} \ddot{x}_{\mathrm{r}}+b_{\mathrm{r}} \dot{x}_{\mathrm{r}}+k_{\mathrm{r}} x_{\mathrm{r}}=A_{\mathrm{r}} P_{\mathrm{c}}-f_{\mathrm{r}} \\
& A_{11}=A_{n,} A_{n} \\
& =\left(A_{12} x_{n \pi}-A_{22} x_{r}\right)-f_{d} \\
& A_{12}=A_{4 \pi} A_{r} \\
& A_{22}=A_{r} A_{r}
\end{aligned}
$$

Mathematical modeling of clutch drive system interaction formula is as follows and we sought to compare and analyze the hysteresis response characteristic result regarding the final clutch pedal effort in accordance with changes in material and length change of oil line [2].

\section{TEST AND RESULTS}

\section{A. Oil Line Test of Varialbe Length}

In order to understand the hysteresis response characteristic of clutch drive system, a test was conducted in accordance with the length change in each of the oil lines via the experiment Jig. When the compressed air pressure of 8bar was consistently supplied to the clutch booster and $0.3 \mathrm{sec}$ and $1.0 \mathrm{sec}$ of pedaling time was applied, a comparison was made of pedal portion pedal effort hysteresis response characteristic in accordance with the change in length in each of the oil line. And cylinder specifications are used in this experiment is as the following Table I.
First, at the time of $0.3 \mathrm{sec}$, the length of oil line was $4 \mathrm{~m}$ and $8 \mathrm{~m}$ in two types and the experiment was conducted by applying each of it to the oil line of a steel material, a plastic material, a rubber material. The pedal load displayed an average of $10 \mathrm{kgf}$ of power conduct up to the point of reaching a maximum of $140 \mathrm{~mm}$ by pressing down the pedal stroke and the hysteresis response characteristic did now show significant difference regarding the change in oil line length. The response characteristic graph in accordance with the change in applicable length is as displayed in Figure III.

TABLE I. SPECIFICATIONS OF MASTER CYLINDER AND BOOSTER

\begin{tabular}{|c|c|c|}
\hline Properties & Master Cylinder & Clutch Booster \\
\hline Mass & $27.56 \mathrm{~g}$ & 628.76 \\
\hline $\begin{array}{c}\text { Diameter } \\
\text { (Inside, Outside) }\end{array}$ & $26 \mathrm{~mm}, 35 \mathrm{~mm}$ & $25 \mathrm{~mm}, 80 \mathrm{~mm}$ \\
\hline Area & $5.31 \times 10^{4} \mathrm{~m}^{2}$ & $4.91 \times 10^{4} \mathrm{~m}^{2}$ \\
\hline Stiffness & $3,000 \mathrm{~N} / \mathrm{m}$ & $1,500 \mathrm{~N} / \mathrm{m}$ \\
\hline
\end{tabular}
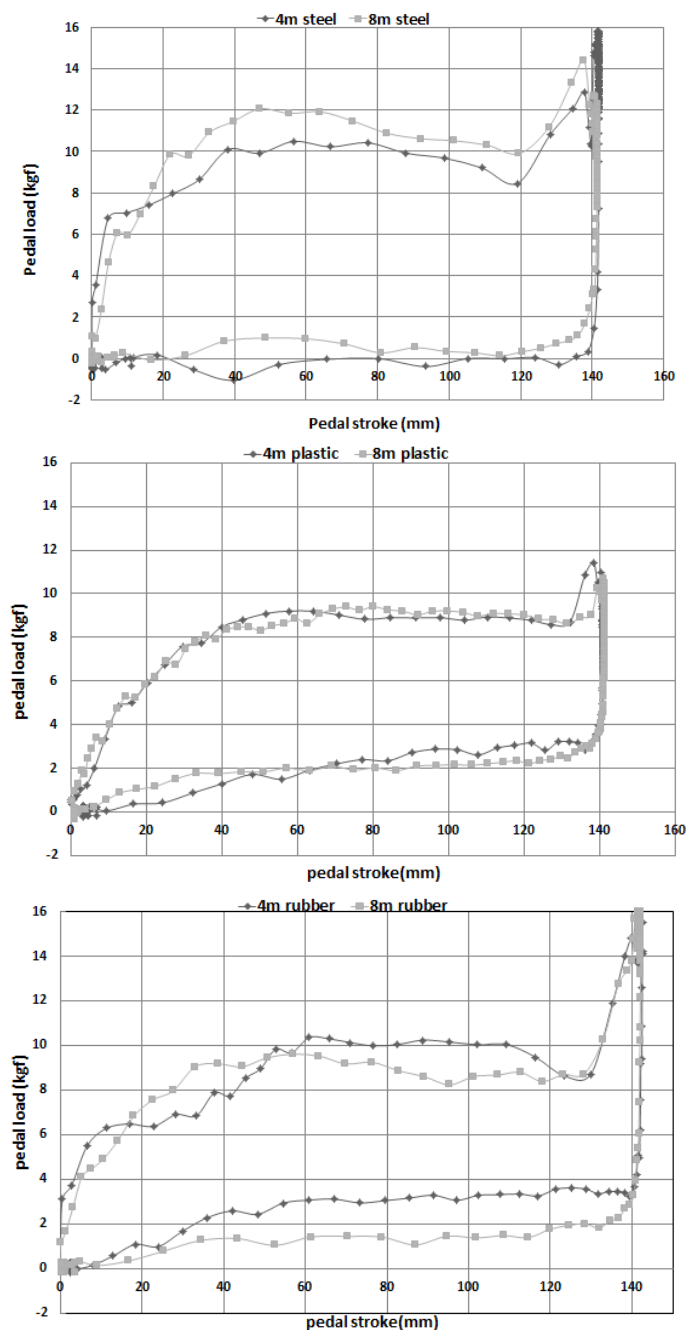

FIGURE III. TEST RESUTLS OF VARIABLE LENGTH FOR 0.3 SECOND 
First, when the effect of materials on the hysteresis characteristics of pedal effort by material length was analyzed at the pedaling duration of 0.3 second and the results were compared, it was known that rubber and plastic did not show difference in hysteresis characteristic by length. But steel showed slightily higher hysteresis behavior in the length of $8 \mathrm{~m}$ than in $4 \mathrm{~m}$. However, it was not statistically significant.

Second, the changes of hysteresis characteristics were analyzed by the length of oil line at the pedaling duration of 1.0 second. Other conditions were the same as the first experiment above 0.3 second.

At the time of 1.0sec, the response characteristic graph in accordance with the change in applicable length is as displayed in Figure IV.
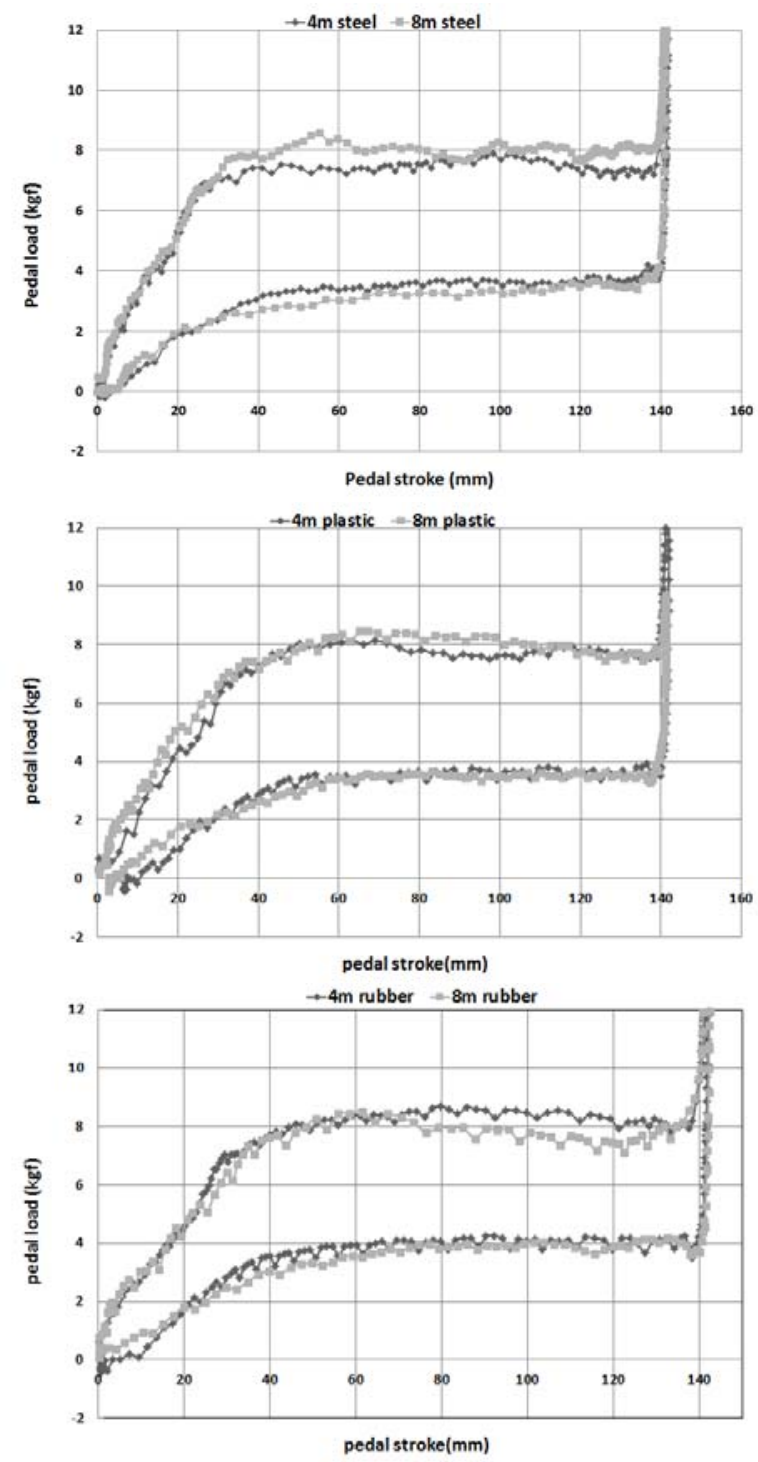

FIGURE IV. TEST RESUTLS OF VARIABLE LENGTH FOR 1.0 SECOND
No difference was found in hysteresis characteristics by length in all three materials for the pedaling duration of 1 second. Like in 0.3 second, only Steel showed insignificantly higher hysteresis behavior in the length of 8 meters than in 4 meters. In addition, when pedaling was given for a short time period of 0.3 second, it was found that pedal load more increased as a whole because the force to the clutch master cylinder increases momentarily than the case of 1 second.

\section{B. Oil Line Test of Variable Materials}

A test in accordance with oil line material change of clutch drive system was conducted. Experiment condition was kept identical in terms of change in length and the experiment condition and $8 \mathrm{~m}$ oil line Steel material, Plastic material, Rubber material oil line was used in comparing pedal portion pedal effort hysteresis response characteristic in accordance with material change.

The result of the experiment displayed the hysteresis response characteristic when steel material oil line was applied, showed higher hysteresis response characteristic compared to the other two materials and the response characteristic graph in accordance with material change is displayed in the following Figure V.
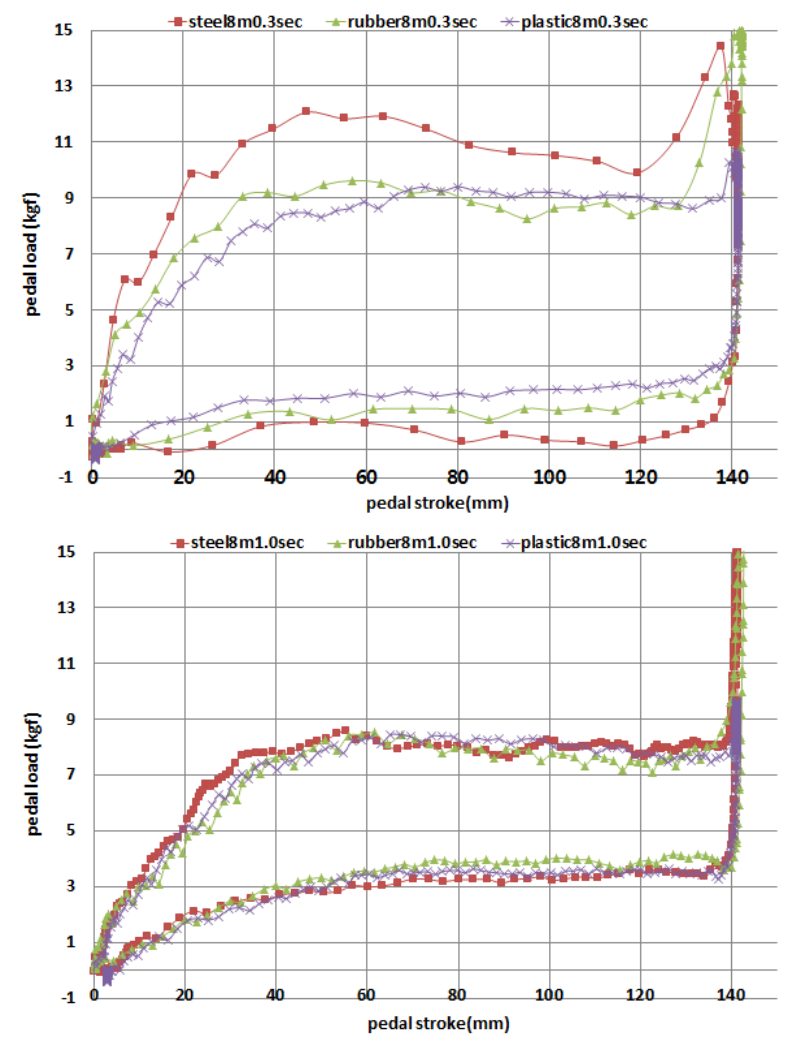

FIGURE V. TEST RESUTLS OF VARIABLE MATERIALS

\section{CONCLUSIONS}

This thesis compared clutch pedal effort hysteresis response characteristic in accordance with changes in oil line length and applied material in clutch drive system. When the 
pedal is pressed, a maximum of $140 \mathrm{~mm}$ displacement arises and in this experiment the strength of the pedal effort of the pedal was shown to be $8-12 \mathrm{kgf}$ and the close curve area which represents hysteresis response characteristic was displayed to be at a similar level.

First, the result of oil line length change showed the effect from change in length in hysteresis response characteristic was inadequate and the oil line length applied to clutch system can differ depending on the size of the automobile in terms of the clutch system actually applied to an automobile. According to these experiment results, response according to the applicable length can also be thought to be inadequate.

Second, from the result of the oil line material change experiment, it was evident that the hysteresis response characteristic was effected by the material and when steel material oil line was applied, the hysteresis characteristic was higher than when plastic and rubber materials were applied.

Furthermore, it turned out hysteresis characteristics by the length of oil line were higher as a whole at the pedaling duration of 0.3 than at 1 second. 0.3 second can be regarded as actual time for a driver to step on a clutch. From such change in characteristic it can be assumed that comparison analysis through special experiment regarding additional detailed variable of clutch drive system will be necessary in the future, as the clutch system itself possesses nonlinear characteristic from various environmental variables even though the change aspect was inadequate compared to the effect of hysteresis of clutch pedal effort in accordance with the auxiliary device applied to the general clutch drive system.

\section{ACKNOWLEDGMENT}

This work was supported by the National Research Foundation of Korea(NRF) Grant funded by the Korean Government(MOE)(No. NRF-2011-0010475)

\section{REFERENCES}

[1] Kenji Imai and Masatoshi Yamada: Pressure Pulsation Transfer in Clutch Hydraulic System, Toyota central Research \& Development Labs.

[2] Tankahisa Hasebe and Hiroshi Yamamoto., "Experimental Study of Reduction Methods for clutch Pedal Vibration and Drive Train Rattling Noise from Clutch System," Society of Automotive Engineers 932007, pp.39-44 